\title{
The Impact of Nutrition on the Autonomic Nervous System
}

\author{
Buchhorn R*, Hauk F${ }^{\S}$, Meint $S^{\S}$ and Willaschek C
}

Caritas Krankenhaus Bad Mergentheim, Department of Pediatrics, Germany

$\S$ This paper contains parts of the doctoral thesis of F.Hauk and S. Meint

*Corresponding author: Prof. Reiner Buchhorn, Klinik für Kinder- und Jugendmedizin, Caritas Krankenhaus Bad Mergentheim, Uhlandstr. 7, 97980 Bad Mergentheim, Germany, Tel: 07931-582301; Fax: 07931-582390; E-mail: reiner.buchhorn@ckbm.de

\begin{abstract}
Autonomic imbalance measured by 24 hour HRV (heart rate variability) analysis is described for overweight children as well as for patients with anorexia nervosa. Autonomic imbalance is a predictor of possible metabolic syndrome, cardiovascular disease and impaired neurodevelopment. In our cross-sectional study we compared HRV parameters for a healthy control group and constitutional thinness with patients with anorexia nervosa and overweight children. We had found that, children with anorexia nervosa had had significantly lower heart rates at night whilst in overweight children significantly higher heart rates. Concomitant anorexia nervosa patients - children with constitutional thinness and the same body mass index (BMI) had a significantly higher SDNN (standard deviation of all RR-intervals) while overweight children had a significantly lower SDNN. Within all groups we had found a strong and significant correlation between SDNN and heart rate at night. The obesity education program KIDS and the in hospital refeeding in anorexia nervosa patients significantly improved heart rate variability. Chi square test show a highly significant interrelationship between changes in BMI and changes in 24 hour heart rate. Micro nutrition and the role of omega-3-fatty supplementation was measured and discussed. Our data were compared with data of undernourished and stunted children from the literature. We concluded that not current BMI but rather metabolic rate predominantly determines intrinsic heart rate. We propose a comprehensive model to explain the impact of nutrition on the autonomic nervous system and discuss the therapeutic consequences.
\end{abstract}

Received Date: May 23, 2016

Accepted Date: June 09, 2016

Published Date: June 13, 2016

Citation: Buchhorn, R., et al. The Impact of Nutrition on the Autonomic Nervous System. (2016) Int J Food Nutr Sci 3(1): 259-274.

DOI: $10.15436 / 2377-0619.16 .942$

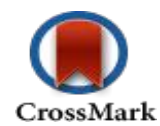

Keywords: Autonomic nervous system; Heart rate variability; Obesity; Metabolic syndrome; Anorexia nervosa; Stunting; Nutrition; Omega-3-fatty acid

\section{Introduction}

Nutrition is a principal dominator of human health. The concept of nutritional transition was developed to explain the changes in the worldwide prevalence of under nutrition and obesity, according to the changes seen over the last decades in lifestyles and standards of living ${ }^{[1]}$. Nutritional related disease burden and mortality is very high: 900 millions of people suffer from under nutrition and 3.5 millions of children. Stunting is the most frequent consequence of under nutrition in 178 millions of children below 5 years. Stunting, low weight and low birth weight, altogether are responsible for 2.2 million deaths among children and $21 \%$ of disability-adjusted life years ${ }^{[2]}$. Growth is considered to be the best global indicator of children's well-being, and growth impairment has both short and long-term consequences ${ }^{[3]}$. There is strong evidence that poor growth within the first 24 months due to malnutrition is associated with delayed mental development, and that there is a relationship between impaired growth status and both poor school performance and reduced intellectual achievement ${ }^{[4]}$. At the same time and in part within the same countries, overweight and 
obesity individuals have reached epidemic proportions, affecting 1.5 billion adults and 200 million children worldwide. Today and even more so in the future we expect a growing prevalence of metabolic syndromes, cardiovascular diseases and diabetes mellitus as consequence of obesity and eating disorders.

Autonomic imbalance measured by 24 hour HRV (heart rate variability) analysis is described for overweight children as well as for patients with anorexia nervos ${ }^{[5]}$. Autonomic imbalance is a predictor for metabolic syndrome $\mathrm{e}^{[\mathrm{f}]}$, cardiovascular disease $\mathrm{e}^{[7]}$ and impaired neurodevelopment. We recently publish an early life stress model to explain the link between poor growth, cardiovascular risk and cognition deficits by a mechanism called "autonomic imprinting"[8]. The environmental conditions that are experienced in early life can profoundly influence human biology and long-term healthy. Early-life nutrition and stress are among the best documented examples of such conditions, because they influence the adult risk of metabolic disease, such as diabetes mellitus and cardiovascular disease.

We now propose a comprehensive model to explain the impact of nutrition on the autonomic nervous system with respect to the underlying nutritional disorders.

\section{Methods}

Fundamental to all HRV data analysis, is a group of successive normal RR intervals in sinus rhythm (so-called NN intervals in comparison to RR intervals in cardiac arrhythmia) in a defined period of time in minutes (so called short-term HRV) or in 24 hours (so-called long-term HRV) ${ }^{[7]}$. The Time Domain Analysis is done by the processing of the data with simple statistical methods. For didactic reasons in this study we focus on the statistical analysis of the following 4 parameters, which are briefly outlined below:

- Heart rate: The easiest but very important HRV parameter is the average sinus rhythm heart rate, since all other parameters are significantly affected by the heart rate.

- SDNN: Standard deviation of all normal RR intervals in a time frame. This global HRV parameter represents the overall variability of the autonomic nervous system.

- $\quad$ RMSSD: Square root of the arithmetic mean of the squared deviation of successive normal RR intervals in a time frame. This parameter is mainly influenced by the parasympathetic nervous system.

- $\quad$ sNN50/pNN50: Absolute number (sNN50) or number of pairs of adjacent NN intervals differing by more than 50ms divided by the total number of all NN intervals (pNN50). This parameter is mainly influenced by the parasympathetic nervous system.

Beat-to-beat fluctuations were transformed to the frequency domain using Fast Fourier Transformation. Spectral power was determined over three frequency regions of interest: Very low frequency (VLF, < $0.04 \mathrm{~Hz}$ ), low frequency $(L F, 0.04-0.15 \mathrm{~Hz})$ and high frequency (HF, $0.15-0.4 \mathrm{~Hz}$ ) with derived LF/HF ratio. LF reflects mostly sympathetic activity, HF vagal tone.

HRV represents the autonomic network, which was initially described by Claude Bernard and more recently by Julian Thayer $^{[0]}$. We would like to emphasize, that by the HRV analysis we do not analyze the heart, but rather the autonomic regulation centers of our brain, especially the limbic system and the hypothalamus. Keeping this in mind, the HRV analysis provides us with a unique insight into our emotional regulatory system, which we otherwise could only detect in functional magnetic resonance imaging (MRI) examinations ${ }^{[10]}$. The major advantage especially for the pediatric population is the possibility of measuring the HRV of the young patients unnoticed, under real life conditions with little costs and without the artificial and expensive conditions of an MRI.

\section{Pathophysiology}

We had showed that the "Big Three" of new morbidities in childhood - obesity, anorexia nervosa and attention deficit disorder - are each characterized by a specific pattern of autonomic imbalance. Looking for the cause of autonomic dysfunction in childhood, we realize that nutrition and micro nutrition have a high impact on $\mathrm{HRV}^{[5]}$.

We performed a cross-sectional study comparing our HRV data from healthy children with a body mass index between $>10^{\text {th }}$ percentile and $<90^{\text {th }}$ percentile and aged between 10 and 16 years, overweight children with a body mass index over the $96^{\text {th }}$ percentile, healthy children with constitutional thinness body mass index lower than the $10^{\text {th }}$ percentile ${ }^{[11]}$ and children with a comparable body mass index due to anorexia nervosa. ANOVA analysis showed significant intergroup differences for heart rate (p $<0.001)$ and SDNN $(\mathrm{p}<0.001)$. Post-hoc testing revealed that healthy thin children had normal heart rates and all in all normal HRV. As shown in figure 1 children with anorexia nervosa had significantly lower heart rates $(\mathrm{p}<0.0001)$ and obese children had significantly higher heart rates $(\mathrm{p}=0.033)$. These differences came along with significantly higher global HRV expressed as SDNN in children with anorexia nervosa $(p<0.0001)$ and significantly lower SDNN in overweight children $(p=0.002)$. However, significantly decreased 24 hour SDNN values in overweight children were a consequence of smaller differences of heart rates between day and night values and this difference in overweight children was mainly derived from elevated heart rates at night. We found the strongest and highly significant relationship between SDNN and heart rate at night with $r=0.891\left(R^{2}=0.79, p<0.001\right)$.

For a better understanding of the interaction between mean heart rate and HRV ${ }^{[12]}$ we have to realize that global HRV depends on heart rate showing a strong correlation during maturation of the autonomic nervous system from infancy to adulthood. Within the same age group between 10 and 16 years, all marks were found to be along on one regression line $\left(r=0.89, \mathrm{R}^{2}=0.79\right)$ but patients with anorexia nervosa, overweight and thin children were clustered ${ }^{[5]}$. 


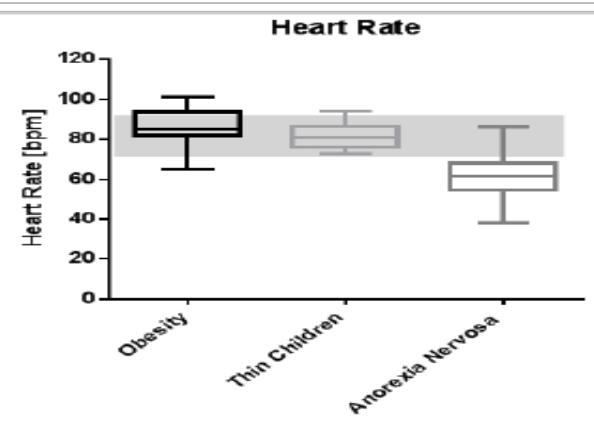

rMSSD
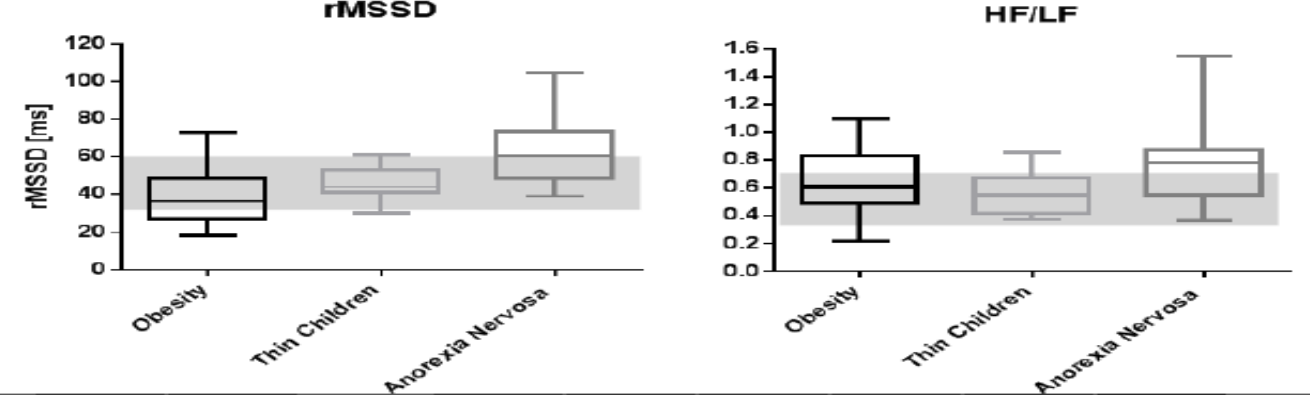

Figure 1: Heart rate variability of children according to their nutritional state

Based upon this strong relationship between heart rate and global HRV (SDNN) we are able to abolish the "abstract world" of HRV analysis and the two dimensional thinking of the autonomic nervous system. Accumulating evidence clearly demonstrates that this assumption is naïve and greatly oversimplifies the complex non-linear interactions between the sympathetic and the parasympathetic divisions of the autonomic nervous system ${ }^{[13]}$. Most of these interpretations are based upon the short time HRV response to interventions that either increase or reduce autonomic neural activity in animal models. Our data now demonstrate the effect of specific interventions on long term HRV and circadian rhythm during the complex maturation of the autonomic nervous system in childhood.

Based upon these data, heart rate regulation in children not only results from changes in cardiac autonomic regulation but also from alterations of the intrinsic pacemaker rate. If one regards metabolic rate as the universal 'pacemaker' for biological processes $^{[14]}$ then metabolism may drive the operating speed of closely related physiological functions (e.g. breathing and heart rhythm). G. Weitz et al. ${ }^{[15]}$ demonstrated that high resting energy expenditure is associated with lower long wave fluctuations (VLF) of heart rate in subjects with normal birth weight; the opposite can be seen in subjects with low birth weight. Both the very low frequency component and the resting energy expenditure decline throughout life time. Further evidence indicates that metabolic rate does not necessarily drive food-intake rate, but conversely increased food intake may elevate metabolic rate. From an evolutionary biologist's perspective, the correlation between caloric intake and heart rate during sleep seems to be part of the physiological regulation during hibernation ${ }^{[16]}$. This relationship has been best studied in the regulation of food intake in mammalian hibernators reviewed by GL Florant et al. in 2011 ${ }^{[17]}$ : “... this form of self-induced anorexia hypothesized that annual fluctuations of food intake in these animals were likely the result of an endogenous 'sliding set-point' mechanism, which sets a different 'ideal' body mass for each season, presumably regulated by hypothalamic control of food intake"[18]. This regulation seems not to be related to the autonomic nervous system but to the hypothalamus-pituitary-adrenocortical connection (HPA axis) ${ }^{[16]}$ and related suprachiasmatic nuclei as circadian clocks ${ }^{[19]}$.

Furthermore, we must take into account three main sub-systems of the autonomic nervous system: the parasympathetic nervous system, sympathetic nervous system, and the "mysterious" enteric nervous system. Depending on the circumstances, these sub-systems may operate independently one another or interact co-operatively. The enteric nervous system can operate autonomously and has the capacity to alter its response depending on such factors as bulk, nutrient composition and the microbiome.

\section{Taken together interpretation of HRV in children has to consider at least 4 qualities}

Intrinsic pacemaker rate: The intrinsic pacemaker rate depends on age and caloric intake and is represented by heart rate, SDNN and Total Power (probably most of all by the very low frequency power). The intrinsic pacemaker rate predicts the baseline heart rate and seems to be related to the metabolic rate and is generated from the hypothalamus. The instantaneous heart rate regulation around this baseline heart rate depends on symapatho-vagal regulation.

Circadian rhythm: The circadian rhythm generated by the suprachiasmatic nuclei and the hypothalamus-pituitary-adrenocortical connection (HPA axis).

Parasympathetic activity: The parasympathetic activity generated from specific nuclei in the central nervous system responsible for the "rest-and-digest" or "feed and breed" response that is represented by RMSSD, pNN50 and high frequency power. According to Porges polyvagal theory ${ }^{[20]}$ the "new vagus" is connected to the frontal cortex and represents social interaction as well as the so called executive functions ${ }^{[21]}$. 
Sympathetic activity: The sympathetic activity (e.g. generated by the limbic system) is responsible for mobilizing the "fight-orflight" response. It is very difficult to estimate sympathetic activity with one HRV parameter, like low frequency power or the HF to LF ratio. However, we feel the quality of "stress" if heart rate is high and HRV is low. The difficulty to define "stress" is one of the most methodological problematic issues in HRV analysis.

\section{Obesity}

Obesity in children today is an important forerunner of cardiovascular risk and is related to a dysfunction of the autonomic nervous system. This hypothesis was confirmed by several publications, recently in a prospective cross-sectional study ${ }^{[22]}$. The pathophysiological pathway from autonomic imbalance and reduced HRV has been attributed to arterial hypertension ${ }^{[23]}$, heart failure $^{[24]}$ and sudden cardiac death ${ }^{[25]}$.

As previously shown, obese children had elevated heart rates and lower SDNN values. The interpretation of these data is much more complicated because this effect cannot be explained by a simple autonomic shift to sympathetic dominance. Time domain analysis showed a parasympathetic withdrawal with lower RMSSD and pNN50. However these trends were not confirmed by the frequency domain analysis. In contrast LF values decreased, HF values were unchanged and the HF/LF ratio increased which has to be interpreted as a shift to parasympathic dominance. All considered significantly higher heart rates at night in overweight children cannot be explained by a simple autonomic shift in HRV analysis toward a sympathetic dominance. Obesity probably increases the intrinsic pacemaker rate indicated by higher heart rates and lower global HRV. The lower Total Power of the Fast Fourier Analysis is related in to a simultaneous decrease in VLF, LF and HF Power.

This model may explain the autonomic effects of the childhood obesity program "KIDS" in 12 children. After a 6 month high intensity education and physical training program, we observed a significant decrease in heart rate (figure 2) and an increase in HRV indicated by SDNN and Total Power although the body mass index decreased only insignificantly. After a further six month low intensity training program the children's HRV decreased once more.

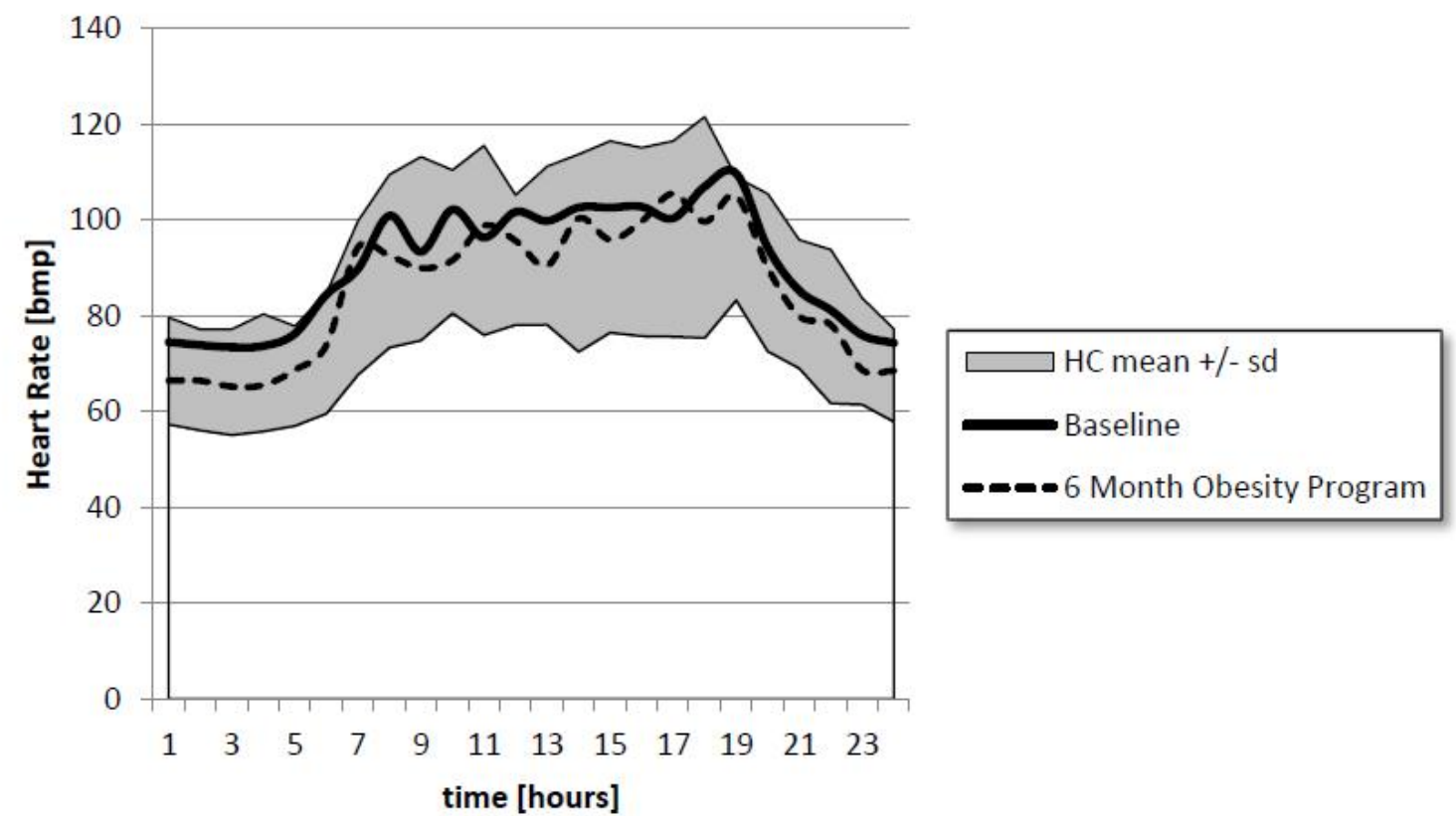

Figure 2: Circadian heart rate of 12 children who suffering from obesity

The Fast Fourier Analysis of 24 hour HRV shows that the significant HRV improvement after 6 month of intensive training was related to the increase of VLF Power with unchanged HF to LF ratio (figure 3). This might be evidence that increased caloric intake leads to a "hypermetabolic state" with higher heart rates at night and lower VLF power, and no clear effect on sympathovagal balance. If autonomic regulation worsened and VLF decreased again - despite the lower body mass indices - a majority of children of our KIDS program probably increased their caloric intake again during the low intensity training during the second half of the one year program.

Our data has further implication for cardiovascular risk stratification in obese children. As shown by Pal et al. ${ }^{[24]}$ a reduced HRV is a sign of pre-hypertension, and moreover, a reduced heart rate day to night ratio is a sign of vascular disease and a risk of all-cause mortality ${ }^{[26]}$. These insights open new therapeutic approaches to improve cardiovascular risk for example by a chrono pharmacotherapy ${ }^{[2]}$ with a beta-blocker to decrease heart rate at night. Furthermore caloric reduction appears to be a very strong interventional tool improving HRV and reducing cardiovascular risk ${ }^{[28]}$. HRV analysis could clearly demonstrate to the patient the success of these interventions long before a BMI change. 


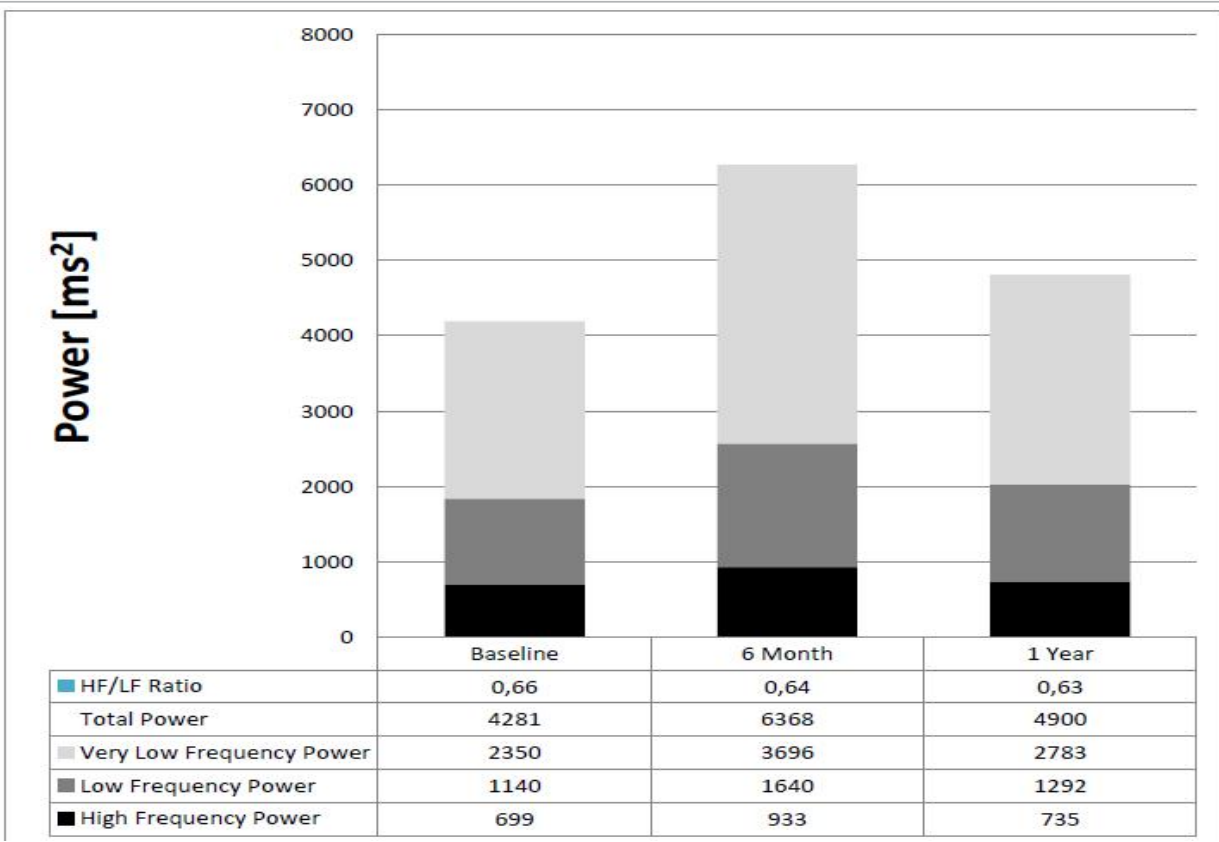

Figure 3: Effect of the childhood obesity program "KIDS" on Fast Fourier Analysis

\section{HRV and the metabolic syndrome}

The criteria for the metabolic syndrome in a child's age have been recently defined for the European IDEFICS study (Identification and prevention of Dietary- and lifestyle-induced health Effects in Children and infants) ${ }^{[29}$. Based on the criteria, namely waist circumference, blood pressure, blood lipids, and insulin resistance, a Monitor Level and an Action Level have been defined as above the $90^{\text {th }}$ percentile and $95^{\text {th }}$ percentile respectively. We examined the criteria of 43 children who were presented to our clinic, in the context of the first examination to enter the obesity training program KIDS and compared the criteria with the HRV in the 24 hour long-term ECG. 11 children were metabolically healthy, 32 children corresponded to the so-called Monitor Level, which included 20 children who presented with an apparent metabolic syndrome (so called Action Level). The classification of the groups was carried out based on the measurement variables and the metabolic parameters in Table 1. None of the mentioned parameters from this chart were predictive for the metabolic syndrome by themselves. A progressive deterioration of the HRV parameters in the group was clearly demonstrated: metabolically healthy > obesity Monitor Level > Action Level. Finally, these 20 obese children who had an apparent metabolic syndrome (Action Level) showed a highly significant decrease with the HRV in the 24 hour longterm ECG (table 1). This decrease affected all frequencies of the Fast Fourier Analysis (figure 4). This figure demonstrates that the changes in the HRV in children with a metabolic syndrome occur especially at night and therefore cannot be directly attributed to physical activity; instead the decrease more likely reflects the neuroendocrine changes during physical inactivity. The calculation of the correlation coefficient (table 2) between the metabolic parameters and the HRV parameters confirm the connection between the heart frequency variability, the arterial hypertension and the glucose metabolism, which were described in adults ${ }^{[30,31]}$. Furthermore, a significant relationship to age is shown which correlates with the observation that the frequency of the metabolic syndrome in obese children increases significantly after the onset of puberty ${ }^{[32]}$. Recently our results have been confirmed in 421 adolescents of the Penn State Children cohort during a prospective study ${ }^{[6,33]}$. The recently published Framingham Heart study shows that autonomous imbalance is the essential predictor for the metabolic syndrome, cardiovascular diseases, diabetes and overall mortality ${ }^{[31]}$. We used this method in the evaluation of the KIDS training program and during the substitution of omega-3-fats and could show significant effects in smaller groups without causing the children pain by taking blood. 
Impact of Nutrition on Nervous System

Table 1: Metabolic- and HRV- parameter related to the metabolic syndrome according IDEFICS criteria

\begin{tabular}{|c|c|c|c|c|c|c|c|c|}
\hline & \multicolumn{2}{|c|}{ Healthy Control } & \multirow{2}{*}{\multicolumn{2}{|c|}{$\begin{array}{c}\text { Obesity } \\
\text { Metabolic healthy }\end{array}$}} & \multicolumn{4}{|c|}{ Obesity with metabolic syndrome } \\
\hline \multirow{3}{*}{ Number of patients } & & & & & Moni & evel & Action & vel \\
\hline & \multicolumn{2}{|c|}{$\mathbf{N}=86$} & \multicolumn{2}{|c|}{$\mathbf{N}=\mathbf{1 1}$} & \multicolumn{2}{|c|}{$\mathbf{N}=\mathbf{1 2}$} & \multicolumn{2}{|c|}{$\mathbf{N}=\mathbf{2 0}$} \\
\hline & Mean & SD & Mean & SD & Mean & SD & Mean & SD \\
\hline Age [years] & 11.2 & 2.7 & 11.1 & 1.8 & 11.5 & 2.4 & 12.2 & 2.8 \\
\hline Waist circumference Perc. [\%] & & & 98.8 & 0.4 & 96.7 & 3.6 & 93.3 & 19.7 \\
\hline Height Perc. [\%] & 46.2 & 27.3 & $68.9^{* *}$ & 23.4 & $67.8^{*}$ & 26.9 & $70.4^{* * *}$ & 26.5 \\
\hline Body Mass Index $\left[\mathrm{kg} / \mathrm{m}^{2}\right]$ & 17.3 & 2.4 & $25.4 * * *$ & 2.0 & $26.4 * * *$ & 4.0 & $30.8^{* * *}$ & 4.9 \\
\hline Body Mass Index SDS & -0.3 & 0.8 & $2.0 * * *$ & 0.3 & $2.2 * * *$ & 0.5 & $2.6^{* * *}$ & 0.5 \\
\hline Systolic Pressure [mmHg] & 114 & 13 & 116 & 11 & 124 & 15 & $132 * * *$ & 14 \\
\hline Diastolic Pressure [mmHg] & 61 & 8 & 62 & 11 & 66 & 9 & 70 & 12 \\
\hline Triglyceride [mg/dl] & \multicolumn{2}{|c|}{$35-180$} & 74.7 & 24.3 & 90.3 & 36,2 & 107.6 & 48.0 \\
\hline Cholesterine [mg/dl] & \multicolumn{2}{|c|}{$0-170$} & 160.7 & 26.4 & 159.7 & 26.0 & 157.8 & 38.9 \\
\hline High Density Lipoprotein [mg/dl] & \multicolumn{2}{|c|}{$35-145$} & 62.8 & 6.5 & 53.0 & 9.0 & 44.47 & 10.7 \\
\hline HOMA & \multicolumn{2}{|c|}{$0,81-1,87$} & 1.50 & 0.50 & 2.11 & 1.60 & 3.90 & 2.41 \\
\hline Heart rate $24 \mathrm{~h}[1 / \mathrm{min}]$ & 81,6 & 8,8 & 85,3 & 6,1 & 86,5 & 7,3 & $91,1 * * * *$ & 6,7 \\
\hline SDNN [ms] & 169 & 45 & 148 & 32 & $139 *$ & 40 & $131 * * *$ & 21 \\
\hline pNN50 [ms] & 26 & 10 & 22 & 10 & 19 & 11 & $15^{* *}$ & 9 \\
\hline rMSSD [ms] & 52 & 16 & 46 & 18 & 40 & 15 & $35 * *$ & 11 \\
\hline Total Power $\left[\mathrm{ms}^{2}\right]$ & 6052 & 2884 & 5118 & 2769 & 3882 & 1756 & $3178 * *$ & 893 \\
\hline VLF 24h [ms²] & 3527 & 2305 & 2979 & 1808 & 2115 & 1125 & $1734 * *$ & 534 \\
\hline LF 24h $\left[\mathrm{ms}^{2}\right]$ & 1595 & 617 & 1334 & 789 & 1074 & 399 & $878^{* *}$ & 299 \\
\hline HF 24h $\left[\mathrm{ms}^{2}\right]$ & 846 & 317 & 714 & 298 & 608 & 326 & $487 * *$ & 210 \\
\hline HF/LF 24h $\left[\mathrm{ms}^{2}\right]$ & 0.56 & 0.19 & 0.59 & 0.23 & 0.55 & 0.22 & 0.56 & 0.19 \\
\hline \multicolumn{9}{|c|}{$\begin{array}{l}\text { *T-test: compared to healthy control } \\
\text { Metabolic parameters are not tested } \\
{ }^{\circ} \text { HOMA: according IDEFICS study., age }<11 \text { years }\end{array}$} \\
\hline
\end{tabular}

Table 2: Correlation coefficients between HRV and metabolic parameters in 43 obese children

\begin{tabular}{|c|c|c|c|c|c|}
\hline & Heart rate & SDNN & RMSSD & Total Power & HF Power \\
\hline Age [years] & -0.15 & $-0.33^{*}$ & -0.29 & -0.26 & $-0.35^{*}$ \\
\hline Waist circumference $[\mathrm{cm}]$ & -0.19 & 0.21 & -0.03 & -0.03 & -0.15 \\
\hline Body Mass Index [kg/m2] & -0.12 & 0.16 & -0.06 & -0.1 & -0.09 \\
\hline Systolic Blood Pressure [mmHg] & 0.19 & -0.29 & $-0.34 *$ & -0.29 & $-0.35^{*}$ \\
\hline Diastolic Blood Pressure [mmHg] & 0.18 & -0.13 & -0.25 & -0.29 & -0.26 \\
\hline Triglyceride [mg/dl] & 0.19 & -0.28 & -0.1 & -0.25 & -0.02 \\
\hline High Density Lipoprotein [mg/dl] & -0.14 & 0.21 & 0.23 & 0.31 & 0.25 \\
\hline Blood glucose [mg/dl] & 0.09 & 0.004 & -0.17 & -0.23 & -0.14 \\
\hline HOMA Index & 0.2 & -0.25 & $-0.38^{*}$ & $-0.37 *$ & $-0.42 *$ \\
\hline
\end{tabular}



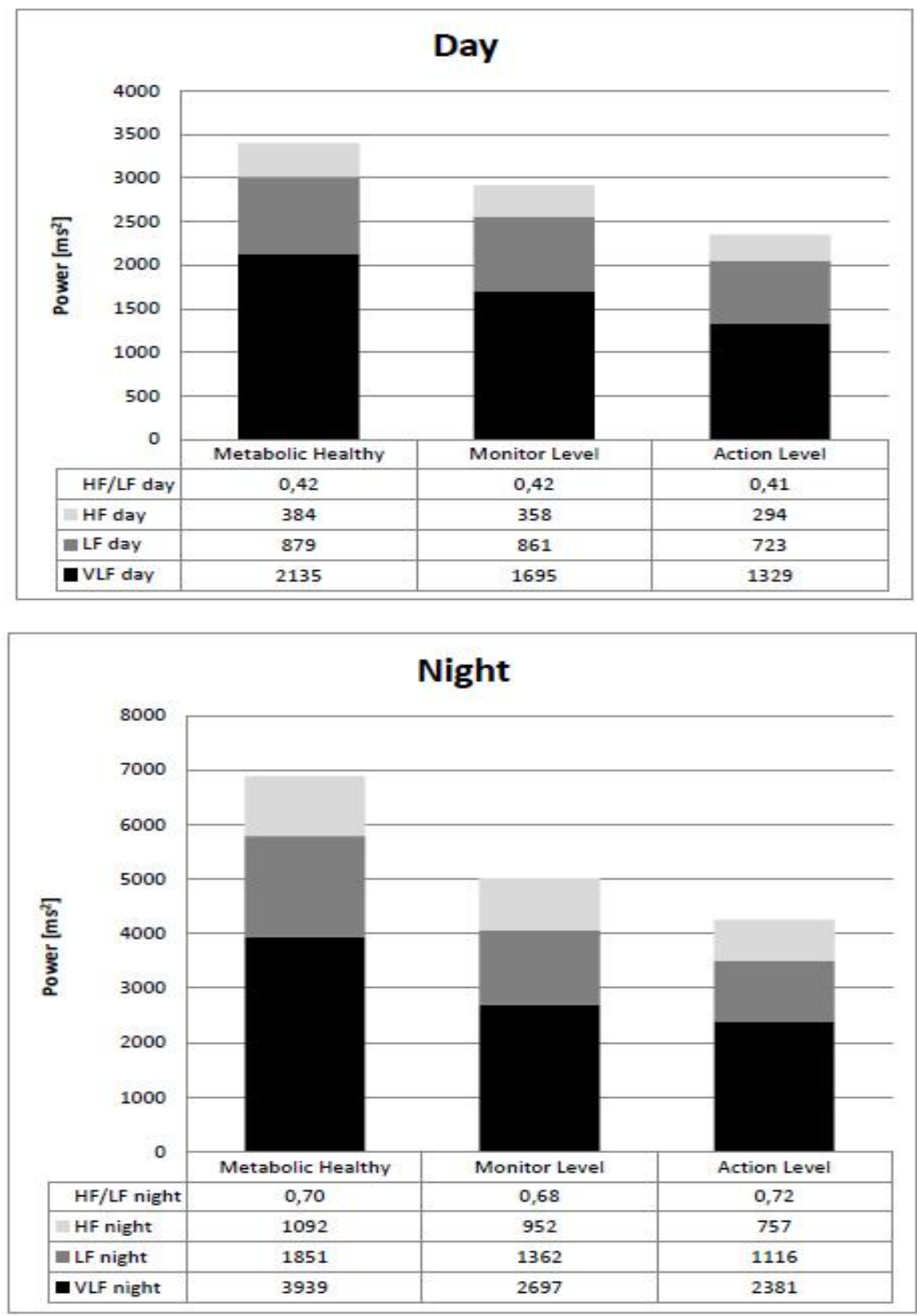

Figure 4: Metabolic syndrome and Fast Fourier Analysis of 24 hour HRV in obese children

\section{Anorexia Nervosa}

Girls with anorexia nervosa suffer from amenorrhea. In contrast to patients with functional normogonadotropic, hypothalamic amenorrhea, patients with amenorrhea due to anorexia nervosa showed a significant dysregulation in multiple HRV parameters $^{[33]}$. The authors suggest that an anorexic biological spectrum including normogonadotropic, hypogonadotropic hypothalamic amenorrhea and anorexia nervosa might exist in adolescents, expressing itself on different levels of severity, including hormonal, autonomic and psychopathological aspects with an origin in the hypothalamic regulation.

Reviews today still show that there is no consistent view regarding the autonomic dysfunction in anorexia nervosa. Our data suggest that current BMI or nutritional state is not directly associated with HRV parameters. We agree at large with Petretta et al. ${ }^{[35]}$ who demonstrated normal HRV parameters in women with constitutional thinness compared to enhanced values in patients with anorexia nervosa. At hospital admission, all children with anorexia nervosa had the well-known parasympathetic dominance with low heart rate, high HRV parameters and a significant elevation of the HF/LF ratio. After one month of re-feeding, HRV decreased to nearly normal values.

At first view low heart rates and higher HRV and Total Power in children with anorexia nervosa may be explained by a simple autonomic shift in HRV analysis toward parasympathic dominance indicated by higher RMSSD, High Frequency Power and HF to LF ratio (Figure 5). However during re-feeding we observed a quick "normalization" of HRV values but the HF to LF ratio remained elevated. Again these autonomic effects during re-feeding may be explained by an increase of the intrinsic pacemaker rate with lower HRV indicated by lower SDNN and lower Total Power. In detail the decrease in Total Power is accompanied by lower HF, LF and VLF but with an unchanged HF to LF ratio (Figure 6). 


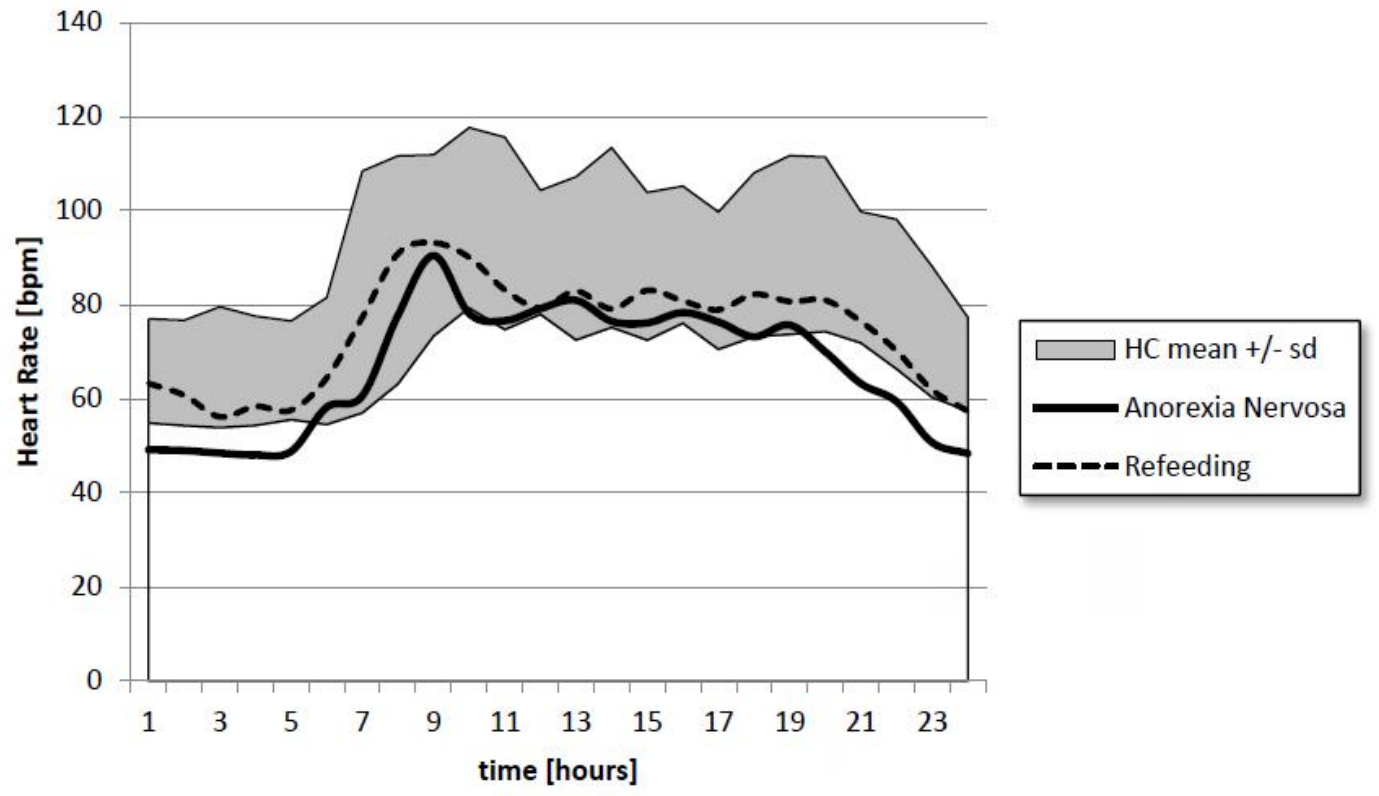

Figure 5: Circadian heart rate of 16 children

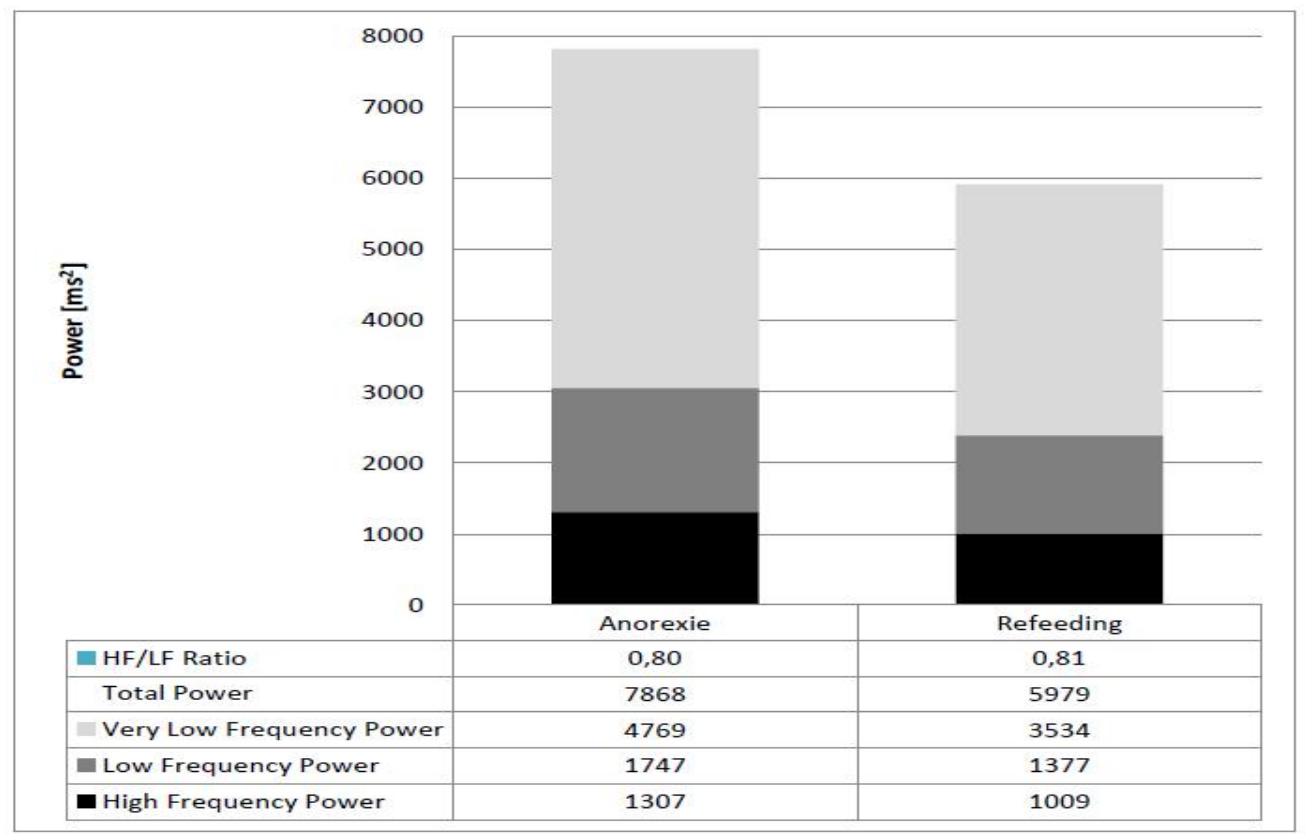

Figure 6: 24 hour Fast Fourier Analysis of 16 children suffering from anorexia nervosa

Our discovery of the rapid reaction of the autonomous nervous system to the increased caloric intake offers a possible explanation of hitherto contradicting results, most previous studies do not account for the caloric intake but only BMI. Given the rapid changes in HRV parameters some previous measurements may have detected the autonomic responses instead of the initial autonomic state of the illness. Moreover, this rapid reaction shows that the autonomous nervous system is not a stable but a very dynamic system. Nonetheless HRV analysis and the known changes in anorexia nervosa are helpful tools that aid in differentiating between ambiguous cases of anorexia nervosa and habitual slimness.

\section{These findings have important therapeutic implications for the treatment of anorexia nervosa}

We modified our therapeutic protocol with caloric and vitamin B supplementation according to the NICE protocol ${ }^{[36]}$ and additional omega-3-acid supplementation. We observed an improved weight gain in 27 children after this change of protocol compared to our own historical data (Figure 7). We also compared our retrospective data with published interventional studies ${ }^{[37]}$ : Weight gain was at its best with exclusively orally fed patients. Weight gain was comparable to the patients with additional nasogastric tube feeding and better than patients with additional parenteral nutrition. In contrast to these interventions none of our patients needed antianxiety medications. 


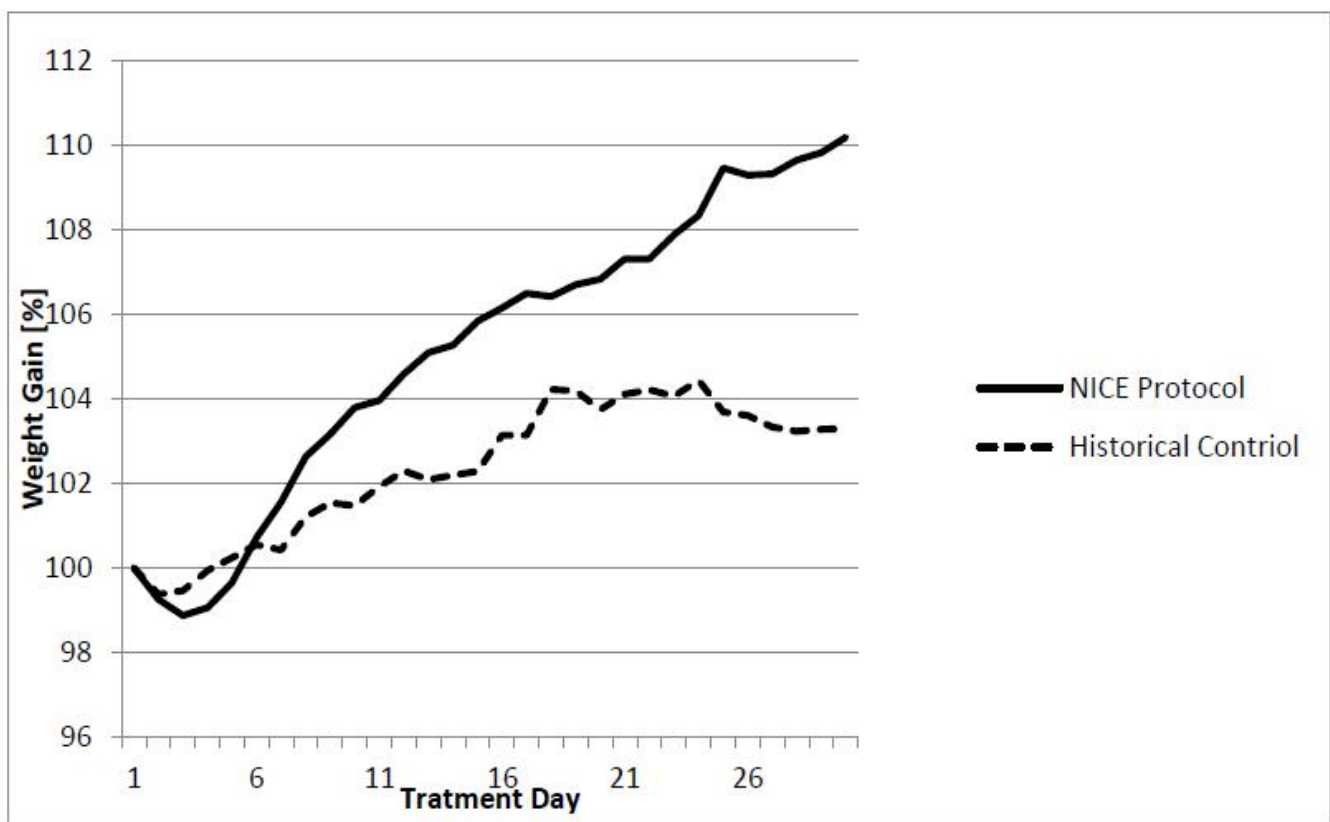

Figure 7: Weight gain of children with anorexia nervosa

A consequent increase of caloric intake at the beginning of hospital stay through high caloric supplementation is probably necessary to reach the "autonomic switch" and interrupt the "hibernating circuit" induced by caloric restriction. However, as shown in figure 8, this "autonomic switch" impairs weight gain after the first treatment week from up to $400 \mathrm{~g} / \mathrm{day}$ to $100 \mathrm{~g} / \mathrm{day}$ by the increase of metabolic and heart rate. In our view the "hibernating circuit" seems to obliterate the emotional memory of the patients, which is reawakening during nutritional rehabilitation. This "flash back" of negative emotions may explain the resistance of the patients against nutrition therapy after reaching the caloric gain of $60 \mathrm{kcal} / \mathrm{kg} / \mathrm{day}$ after the first treatment week. We attempted to weaken this "autonomic switch" to sympathetic dominance with omega 3 fatty acid supplementation ${ }^{[38]}$ and biofeedback training ${ }^{[39]}$. Routine supplementation of omega-3-fatty acids, accompanied by sport ${ }^{[40]}$ and possibly beta-blockers in patients with extreme heart rate increases may help to smooth the "autonomic switch" and help the patient to tolerate the loss of the "vagal brake".

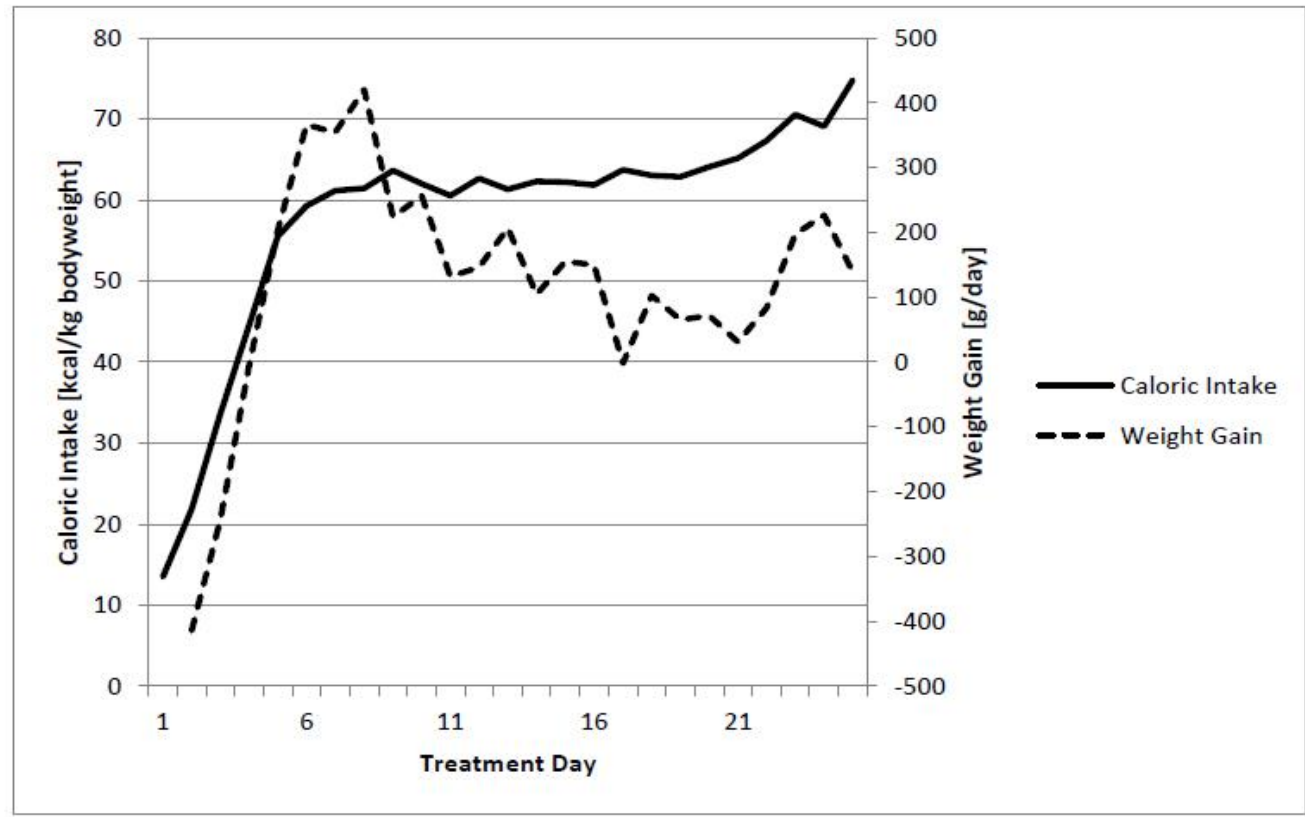

Figure 8: Daily weight gain and caloric intake

We observed reproducible autonomic changes in patients with Anorexia Nervosa during follow up. During the initial state of starvation, HRV was very high throughout the day and night. During successful re-feeding the patients showed a sympathetic shift to normal values at day. During longtime follow up with multiple hospital stays most patients showed a loss of vagal activity at day and in part high HRV at night. This "autonomic scare" has been described by M. Lachish et al. ${ }^{[41]}$, but we are not sure whether this sympathetic regulation was not the cause of the disease prior to starvation. In the sense of a learning model the children had learned to treat their emotional stress by starvation. We are deeply convinced that Anorexia Nervosa is preventable by the consequent treat- 
ment of emotional stress and autonomic dysfunction in children for example after traumas (sexual abuse, familial conflicts) or a genuine stress diseases like attention deficit disorder.

\section{HRV and Micro Nutrition}

Omega 3 fatty acids consistently produce statistically significant reductions in baseline heart rate that are accompanied by increase in $\mathrm{HRV}^{[42]}$. Recent data strongly suggest that intrinsic changes in pacemaker rate may play a more important role in omega 3 fatty acid induced heart rate reductions than do changes in cardiac autonomic regulation.

There is increasing evidence that supplementation of omega 3 fatty acids have beneficial effects in children with cognitive disorders like $\mathrm{ADHD}^{[43]}$. Further evidence exists about the beneficial effects in adults with arrhythmias ${ }^{[4,45]}$. Cardiovascular risk in children with small gestational age depends on nutritional intake of omega-3-fatty acids ${ }^{[46]}$.

We routinely use omega-3-fatty acid supplementation in children with autonomic imbalance due to metabolic syndrome, growth failure or during refeeding of children with anorexia nervosa. We used different preparations with $1-2 \mathrm{~g}$ fish oil per day. As shown in the table 3, there were highly significant effects on heart rate and HRV. If most of these children with metabolic syndrome and growth failure had elevated heart rate we interpret a normalization of heart rates as a beneficial effect.

In detail, the highly significant heart rate effects of omega-3-fatty acids seem to be mediated by vagal activity if RMSSD showed highly significant changes which might explain the changing heart rates. However, Fast Fourier Analysis showed an unchanged HF to LF ratio and indicated a heart rate effect independent of sympathovagal balance.

Taken together omega 3 fatty acids seem to have highly significant effects on intrinsic pacemaker rate and HRV in children with autonomic disorders that may be related to their beneficial clinical effects.

Table 3: Absolute difference of 24 hours HRV-parameters before and after 3 month supplementation with omega-3-fatty acids

\begin{tabular}{|c|c|c|c|}
\hline Parameter & Obesity & Growth Failure & p-value: Obesity + Growth Failure \\
\hline $\mathbf{N}$ & 10 & 16 & 26 \\
\hline Heart Rate [bpm] & $-5.7 * *$ & $-7.7 * *$ & $<0.0001$ \\
\hline SDNN [ms] & +14.0 & +15.7 & 0.015 \\
\hline RMSSD [ms] & $+5.1^{*}$ & $+8.5^{*}$ & 0.0003 \\
\hline $\mathbf{T P}\left[\mathrm{ms}^{2}\right]$ & $+1249 *$ & +2205 & 0.003 \\
\hline $\mathrm{VLF}\left[\mathrm{ms}^{2}\right]$ & $+816^{*}$ & +1429 & 0.003 \\
\hline $\mathbf{L F}\left[\mathrm{ms}^{2}\right]$ & $+275^{*}$ & +548 & 0.018 \\
\hline $\mathbf{H F}\left[\mathrm{ms}^{2}\right]$ & +156 & $+214 *$ & 0.0023 \\
\hline HF/LF & +0.1 & -0.02 & ns \\
\hline
\end{tabular}

SDNN: Standard deviation of all NN intervals; RMSSD: The square root of the mean of the sum of the squares of differences between adjacent NN intervals; TP: Total Power VLF: Very low frequency power; LF: Low frequency power HF: High frequency power; HF/LF: Ratio HF to LF. Paired T-test before and after supplementation with omega-3-fatty acids: $<0.0001$

\section{HRV and Environmental Toxins}

The interplay between fish consumption and mercury exposure most of all in pregnant women is complicated. Mercury exposure during childhood seems to affect HRV among Nunavik Inuit children of school age ${ }^{[47]}$. However, the FDA's new advice conclude that pregnant and nursing women should be advised to eat fish to benefit from naturally occurring omega 3 fatty acids, to avoid fish with high levels of mercury and other contaminants, and, if possible, to choose fish with high levels of omega 3 fatty acids $^{[48]}$. Nevertheless other environmental toxins seem to be more important than mercury from sea food:

Bisphenol A (BPA) is a high-volume production chemical with in impact on HRV. Urinary BPA concentration reduces RMSSD and is related to hypertension ${ }^{[49]}$.

Several studies report on the association between short term exposure to ambient air pollution and HRV. An increase in particulate matter with aerodynamic diameter of $10 \mu \mathrm{m}$ or less (PM10) or $2.5 \mu \mathrm{m}$ or less (PM2.5) is associated with a significant reductions in time-domain and in frequency-domain measurements (SDNN and RMSSD ${ }^{[50]}$. The Multi-Ethnic Study of Atherosclerosis showed that autonomic dysfunction may be a mechanism through which particulate air pollution affects cardiovascular risk, especially among persons with metabolic syndrome ${ }^{[51]}$. Occupational exposure to environmental tobacco smoke may decrease heart rate variability ${ }^{[52]}$.

There is growing evidence that intensity of information and communication technology (ICT) usage in childhood have an effect on quality of sleep and HRV ${ }^{[3,54]}$. Means, adjusted for gender, age and BMI of SDNN (99.05 versus $\left.114.12 \mathrm{~ms}, \mathrm{p}=0.002\right)$ and RMSSD (69.87 versus $82.61 \mathrm{~ms}, \mathrm{p}=0.008)$ are significantly lower in high ICT users compared to low ICT users $(\mathrm{N}=61)$.

All things considered particulate air pollution and intensity information and communication technology usage seems to be the most important environmental "toxin" to reduce HRV in childhood. Omega-3 fatty acid supplements offer protection against the adverse cardiac and lipid effects associated with air pollution exposure ${ }^{[55]}$. 


\section{HRV and Stunting}

Recently we published our early life stress model to explain growth failure in children with growth hormone deficiency and children small for gestational age. In these children growth failure is related to $\alpha 2$-adrenoreceptor sub sensitivity, measured by HRV analysis during clonidine testing for growth hormone stimulation ${ }^{[8]}$. Moreover, there is an interaction between neurologic development and HRV: Recently we showed that attention deficit hyperactivity disorder (ADHD) is characterized by reduced vagal tone measured by HRV analysis ${ }^{[56]}$.

Stature- associated polymorphisms using genome-wide data from 253.288 individuals had identified 697 variants at genome-wide significance, that together explained one-fifth of the heritability for adult height ${ }^{[57]}$. Epigenetic heredity thus appears to be a further important determinant of adult height ${ }^{[58]}$. Modulation of DNA methylation is candidate to mediate environmental influence on epigenetic traits. Human height is one of the best defined phenotype and growth failure seems to be a model for imprinting probably due to epigenetic mechanisms.

In 2010,171 million children under 5 years of age were estimated to be stunted globally, with $98 \%$ being from low- and middle-income countries. The understanding of child growth patterns is critical for development of appropriate interventions and their evaluation.

We now compare the data of patients with anorexia nervosa, cachexia due to severe disease, small gestational age and growth failure with data from wasting children caused by malnutrition and stunting from the literature. Stunting is more than simply undernutrition ${ }^{[59]}$. The co-existence of low body weight and/or stunting and obesity in poor populations show that not only caloric intake but also micro nutrition influence growth. In a study from Brazil ${ }^{[60]}$ among stunted women, energy consumption was shown to have no relation to nutritional status, as similar energy consumptions were found for undernourished and obese women, which were $4527 \mathrm{~kJ}$ and $4686 \mathrm{~kJ}$, respectively.

Table 4: Neuroendocrine and HRV parameters in children with different causes of undernutrition or growth failure

\begin{tabular}{|c|c|c|c|c|c|c|}
\hline & \multicolumn{3}{|c|}{ Wasting } & \multirow{2}{*}{$\begin{array}{c}\text { Fetal } \\
\text { SGA }\end{array}$} & \multicolumn{2}{|c|}{ Growth } \\
\hline & Under nutrition & Anorexia Nervosa & Cachexia & & Stunting & Growth Failure \\
\hline \multicolumn{7}{|c|}{ Heart Rate Variability } \\
\hline Heart Rate & $\uparrow$ & $\downarrow$ & $\uparrow$ & $\uparrow$ & $\uparrow$ & $\uparrow$ \\
\hline SDNN & & $\uparrow$ & $\downarrow$ & $\downarrow$ & & $\downarrow$ \\
\hline RMSSD & & $\uparrow$ & $\downarrow$ & $\downarrow$ & & $\downarrow$ \\
\hline pNN50 & & $\uparrow$ & $\downarrow$ & $\downarrow$ & & $\downarrow$ \\
\hline HF & & $\uparrow$ & $\downarrow$ & $\downarrow$ & & $\downarrow$ \\
\hline $\mathbf{L F}$ & & $\leftrightarrow$ & $\downarrow$ & $\leftrightarrow$ & & $\downarrow$ \\
\hline VLF & & $\uparrow$ & $\downarrow$ & $\leftrightarrow$ & & $\leftrightarrow$ \\
\hline LF/HF ratio & $\uparrow$ & $\downarrow$ & $\leftrightarrow$ & $\leftrightarrow$ & & $\leftrightarrow$ \\
\hline HF nu & $\downarrow$ & $\uparrow$ & & & & \\
\hline LF nu & $\uparrow \uparrow$ & $\downarrow$ & & & & \\
\hline \multicolumn{7}{|c|}{ Nutritional Hormones } \\
\hline Leptin & $\downarrow$ & $\downarrow$ & $\downarrow$ & $\downarrow$ & $\downarrow$ & \\
\hline Adiponectin & $\downarrow$ & $\uparrow ?$ & $\uparrow$ & $\downarrow$ & & \\
\hline Ghrelin & $\uparrow$ & $\uparrow$ & & & & \\
\hline \multicolumn{7}{|c|}{ Hypothalamus Hypophysis Axis } \\
\hline GH & $\uparrow$ & $\uparrow$ & & & & \\
\hline IgF 1 & $\downarrow$ & $\downarrow$ & $\downarrow$ & $\downarrow$ & $\downarrow$ & $\downarrow$ \\
\hline CRH/ACTH & $\uparrow$ & $\uparrow$ & & $(\uparrow)$ & $\uparrow$ & $\uparrow$ \\
\hline Cortisol & $\uparrow$ & $\uparrow$ & & $(\uparrow)$ & $\uparrow$ & $\uparrow$ \\
\hline \multicolumn{7}{|c|}{ Cardiovascular Hormones } \\
\hline BNP & $\uparrow$ & & $\uparrow$ & $\uparrow$ & & $(\uparrow)$ \\
\hline Norepinephrine & $\uparrow$ & $(\downarrow)$ & & $\uparrow($ girl $)$ & $\uparrow$ & \\
\hline RAAS & & & & $\uparrow$ (boys) & $\uparrow$ & \\
\hline \multicolumn{7}{|c|}{ Risk Stratification } \\
\hline CV Risk & $\uparrow$ & & $(\uparrow)$ & $\uparrow$ & $\uparrow$ & $\uparrow$ \\
\hline Metabolic Risk & $\uparrow$ & & $(\uparrow)$ & $\uparrow$ & $\uparrow$ & $\uparrow$ \\
\hline Cognitive risk & $\uparrow$ & & $(\uparrow)$ & $\mathrm{ADHD} \uparrow$ & $\uparrow$ & $\mathrm{ADHD} \uparrow$ \\
\hline Obesity risk & & $\downarrow$ & $\downarrow$ & $(\uparrow)$ & $\uparrow$ & $\uparrow$ \\
\hline
\end{tabular}


Current research focuses on neuroendocrine parameters and found altered function in the growth hormone-insulin-like growth factor axis and hypothalamus-hypophysis-axis as shown in table 4. However, low insulin-like growth factor 1 levels and hypersecretion of corticotropin releasing hormone $(\mathrm{CRH})$ and adrenocorticotropic hormone (ACTH) seems to be a uniform picture in wasting and stunting ${ }^{[5]}$. Others found an activated renin angiotensin aldosterone system and changes in the stress response system to explain hypertension and the increased cardiovascular risk ${ }^{[6]]}$.

We focus on the autonomic nervous system and realize that the hypothalamus is connected to the autonomic nervous system and the main brain structures of the limbic system. Using HRV analysis we are abandoning the static biochemical measurements and using the dynamic measurement with respect to circadian rhythms: Unfortunately there is only little information regarding heart rate in stunting. However, LC Fernald et al ${ }^{[62]}$ clearly shows higher resting heart rates of $90.8 \pm 10.8$ beats $/ \mathrm{min}$ in 10 years old stunted children together with significantly enhanced urinary norepinephrine levels. These data are in good accordance with our own HRV data, measured in 101 children with a height below the third percentile. All things considered sympathetic activation seems to be the dominant regulation disorder in all children with growth failure and may explain the enhanced cognitive and cardiovascular risk in later life. Wasting seems to be much more complicated. There seems to be a big difference between anorexia nervosa ${ }^{[63]}$ and undernutrition. As expected, undernutrition is dominated by sympathetic activation ${ }^{[64]}$, but according to the very low caloric intake children with anorexia nervosa clearly show parasympathetic activation ${ }^{[5]}$. This difference is accompanied by low adiponectin levels in undernourished children and probably elevated adiponectin in anorexia nervosa. The cardiovascular risk is enhanced during acute anorexia nervosa but probably not in later life. Caloric restriction is one of the most effective tools for cardiovascular protection ${ }^{[28]}$ in contrast to undernutrition which seems to enhance the cardiovascular risk ${ }^{[59]}$. The main difference between the three wasting syndromes seems to be energy expenditure. The brazil group demonstrated that there was no significant difference in total energy expenditure between undernourished/stunted children compared to non-stunted subjects ${ }^{[6]}$. Children with cardiac cachexia have elevated total energy expenditure.

In contrast to metabolomics analysis, HRV analysis is able to differentiate between undernutrition and anorexia nervosa although both showed evidence for HPA axis activation with elevated CRH/ACTH/cortisol values and suppressed leptin and insulin-like growth factor 1. Moreover, HRV suppression seems to predict an adverse cognitive outcome and the enhanced cardiovascular risk. In contrast, growth seems to be predicted by HPA axis activation and insulin-like growth factor 1 suppression, if children with anorexia nervosa suffer from growth failure to ${ }^{[66]}$. Unfortunately the stress axis is not well investigated in most of the metabolic studies.

The question is why undernourished/stunted children are not able to reduce total energy expenditure and heart rate like children with anorexia nervosa do. Interestingly, providing small-quantity lipid based nutrition supplements significantly increased growth and reduced stunting, wasting and anemia prevalence in young burkinabe children ${ }^{[67]}$. We speculate that supplementation of small-quantity lipid based nutrient with omega three fatty acids (linoleic acid) reduce heart rate and total energy expenditure. This observation clearly showed the crucial need for an understanding of the impact of nutrition and micro nutrition on the autonomic nervous system to improve public health policies in the developing world ${ }^{[68]}$.

\section{Body weight regulation and autonomic nervous system}

After analyzing different nutritional interventions in adolescents with obesity and anorexia nervosa it seemed to be possible to create and verify a model for regulation of body weight in the dimensions of the autonomic nervous system. It became clear that it was not the BMI itself, but rather the changes of the BMI or perhaps the calorie intake that have a highly significantly effect on the autonomic nervous system. In this way we analyzed the changes of two long-term ECGs, each before and after a nutritional intervention. The first group consists of patients with anorexia nervosa $(\mathrm{N}=26, \mathrm{BMI}=15 \pm 1 \mathrm{~kg} /)$, who were treated with a four-week stationary confinement within the hospital with the aim of gaining weight. These patients gained between 2,5 and $5 \mathrm{~kg}$. In a second group we analyzed the changes during the first 6 months of the obesity education program KIDS or a diet advice together with omega 3 fatty acids especially for obese children with a metabolic syndrome, who could not actively take part in the education program because they lived too far away $(\mathrm{N}=38)$. We expected an improvement in the important risk factors due to the Omega-3-fatty acids substitution ${ }^{[69,70]}$.

The distribution of the changes of BMI and HRV are illustrated for the mean heart rate in Figure 9. There is a noticeable cluster formation in the children we examined. As we had expected, the BMI of children with anorexia nervosa increased mainly during the stationary nutritional intervention during which, the autonomous changes in the heart frequency were evident. Diametrical to the heart frequency decrease are the changes in obese children, who actually lowered their BMI. Comparable changes could be seen due to the substitution of Omega-3-fatty acids. However, several children continued to gain weight throughout the obesity training program KIDS, as well as during the substitution of Omega-3-fatty acids (treatment failure).

For the statistical analyzes to proof the interrelationship between weight gain and HRV we speculate that the qualitative changes of BMI are the most important to describe the pathophysiological state as an anabolic (BMI increase) or katabolic (BMI decrease) state. We use fourfold contingency table for each HRV parameter with respect to BMI increase or decrease of the 64 matched pairs. The results of the Chi-square test with Yates' correction for continuity are illustrated in table 5 together with Pearson's coefficients that reflect the strength of the association in the contingency tables. P-values are given: Values of $\mathrm{p}<0.05$ are statistically significant and $p<0.001$ are highly statistically significant: There is absolutely no doubt that the changes of BMI and heart rate are statistically associated. However, what is the autonomic reason of this metabolic induced change of heart rate? The changes of RMSSD, pNN50 and high frequency power suggest a change of autonomic balance in direction of vagal dominance but if LF power changes in the same direction as HF power the LF to HF ratio as a parameter of autonomic balance remained unchanged. We are 
$\overline{\text { concluding that metabolic rate directly influences intrinsic heart rate without changing the autonomic balance. However of cause }}$ mathematic reasons higher heart rates suggest sympathetic dominance and lower heart rates suggest parasympathetic dominance in HRV analyses.

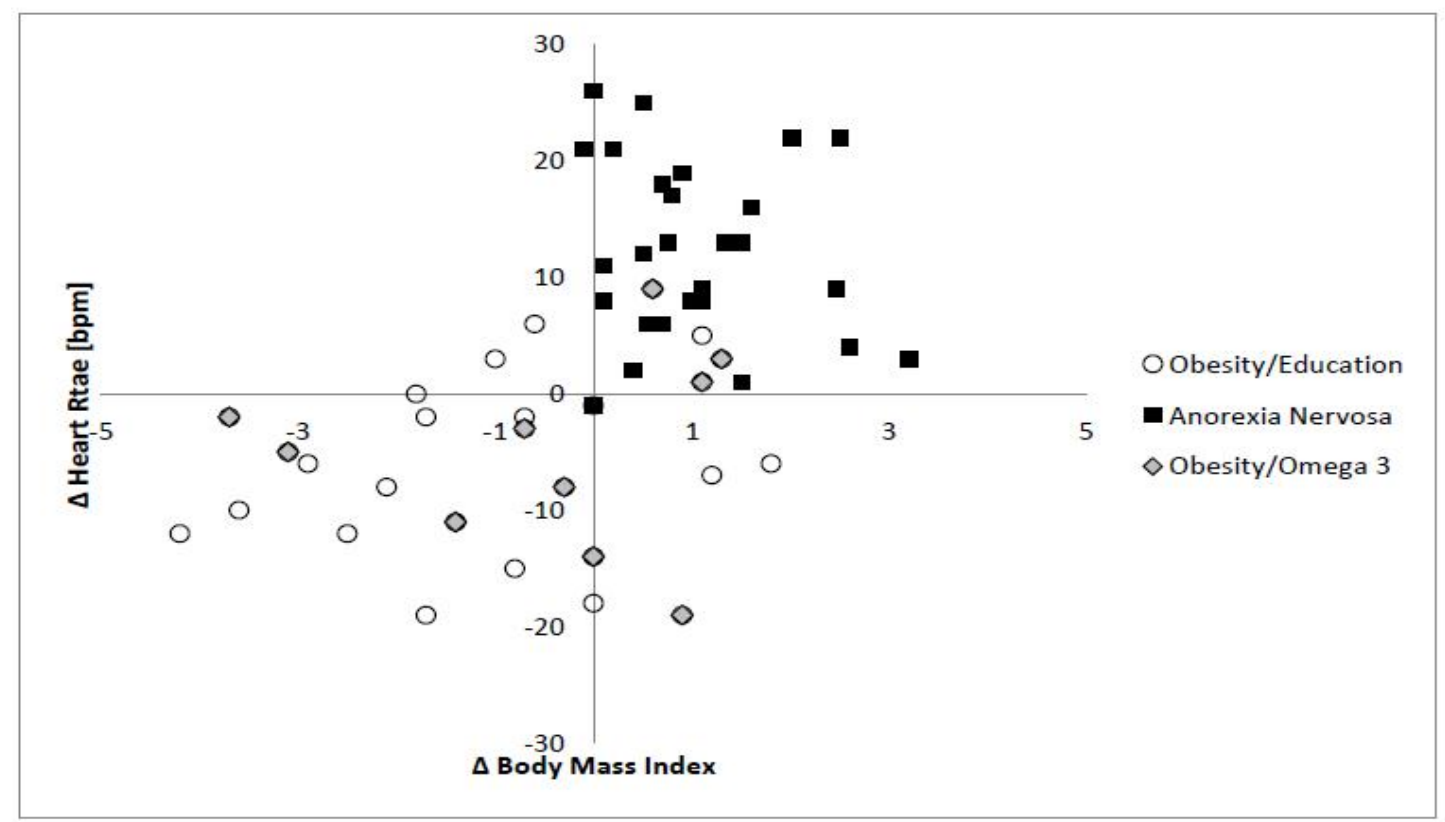

Figure 9: Autonomic effects of nutrition interventions

Table 5: Chi-square analyses of the qualitative effects of BMI changes on HRV analysis in 64 children before and after a nutritional intervention(Obesity $\mathrm{N}=38$; Anorexia nervosa $\mathrm{N}=26$ ), Only significant results are illustrated

\begin{tabular}{|l|c|c|c|}
\hline \multicolumn{1}{|c|}{ Parameter } & Yates' chi-squared test & Pearson R & Significance \\
\hline Heart rate 24h & 0.000 & 0.582 & 0.000 \\
\hline Heart rate Night & 0.000 & 0.522 & 0.000 \\
\hline Heart rate Day & 0.001 & 0.43 & 0.000 \\
\hline HFPower 24h & 0.011 & -0.348 & 0.000 \\
\hline HFPower Day & 0.036 & -0.295 & 0.001 \\
\hline LF Power 24h & 0.003 & -0.402 & 0.000 \\
\hline LF Power Day & 0.001 & -0.434 & 0.004 \\
\hline Max. Heart rate & 0.009 & 0.355 & 0.000 \\
\hline Min. Heart rate & 0.000 & 0.483 & 0.032 \\
\hline pNN50 24h & 0.005 & -0.385 & 0.01 \\
\hline pNN50 Night & 0.02 & -0.321 & 0.001 \\
\hline RMSSD & 0.002 & -0.418 & 0.007 \\
\hline SDNN & 0.016 & -0.335 & 0.000 \\
\hline SD Day & 0.001 & -0.46 & 0.002 \\
\hline Triangle Index Day & 0.005 & -0.385 & 0.000 \\
\hline Total Power 24h & 0.001 & -0.448 & 0.000 \\
\hline Total Power Day & 0.001 & -0.434 & 0.001 \\
\hline VLF Power 24h & 0.003 & -0.402 & 0.01 \\
\hline VLF Power Night & 0.02 & -0.321 & \\
\hline
\end{tabular}

When considering our results it becomes clear, that a higher food supply not only leads to obesity but also to an increase in heart rate and possibly a higher basic metabolic rate. Reducing the food supply led to a decrease in the heart rate and the basic metabolic rate. This happens in order to optimally utilize the calories available and to prevent uncontrolled weight gain or loss according to the actual food supply. However, these changes in the autonomic regulation are accompanied with emotional changes which change the behavior of the children and must be interpreted as therapy obstacles, especially in terms of eating disorders:

Regarding the re-feeding of children with anorexia nervosa our observations show that the calorie substitution primarily leads to rise in heart rate and a need to move, which is difficult to control. We also observed that an increase in body weight could 
only be reached through an excessively high calorie intake of at least $60 \mathrm{kcal} / \mathrm{kg} /$ day (Figure $8 \mathrm{~b}$ ). During the re-feeding stage a rise in the thermogenesis was measured in patients with anorexia nervosa ${ }^{[7]}$, which can further explain the high need for calories. The untrained digestive system of these children cannot adequately deal with the large change in food consumption, which leads to well-known food-intake conflicts. Our systematic investigations confirm the results of studies such as the Minnesota Starvation Experiment of $1944^{[72]}$. This study describes psychopathological changes during a starving period, as well as the high need of calories during the rehabilitation stage in healthy volunteers under experimental terms and conditions.

The same autonomous effect is probably the cause of unsuccessful treatment of obesity. Due to the drop in heart rate and the basic metabolic rate during calorie restriction, the desired weight loss goal was not reached.

The pathophysiological model, which we introduced, offers a first starting-point for the solution to the intensively discussed crucial question of the nutritional physiology. This is why over- or under-feeding leads to individual changes in weight ${ }^{[73]}$; to summarize: where have the calories gone? This question has caused not only scientists but also doctors and patients to feel desperate and distrust one another. The answer to this question is probably the basis for a successful nutritional therapy for weight regulation.

In this sense, we are working on therapeutic interventions in order to stop the vicious circle of metabolic adaptation to weight fluctuations. The routine use of Omega-3 fatty acids within the re-feeding of patients with anorexia nervosa has been very successful so far ${ }^{[38,74]}$. However, the use of Omega-3 fatty acids is not enough to help patients with long and traumatic hospital stays due to anorexia nervosa.

It seems that children with a significant increase of heart frequency during the re-feeding profit especially through with use of beta-blocker therapy. Under the protective effect of the beta-blockers the intake of calories needed to maintain or gain weight becomes lower.

Acknowledgment: We thank Dena Rachdan, medical student University of Niscosia, Turkey, for editorial support.

\section{Reference}

1. Poskitt, E.M. Countries in transition: underweight to obesity non-stop? (2009) Ann Trop Paediatr 29(1): 1-11.

2. Black, R.E., Allen, L.H., Bhutta, Z.A., et al. Maternal and child undernutrition: global and regional exposures and health consequences. (2008) Lancet 371(9608): 243-260.

3. Dewey, K.G., Begum, K. Long-term consequences of stunting in early life. (2011) Matern Child Nutr 7(3): 5-18.

4. Martorell, R., Horta, B.L., Adair, L.S., et al. Weight gain in the first two years of life is an important predictor of schooling outcomes in pooled analyses from five birth cohorts from low- and middle-income countries. (2010) J Nutr 140(2): 348-354.

5. Dippacher, S., Willaschek, C., Buchhorn, R. Different nutritional states and autonomic imbalance in childhood. (2014) Eur J Clin Nutr 68(11): 1271-1273.

6. Rodriguez-Colon, S.M., He, F., Bixler, E.O., et al. Metabolic syndrome burden in apparently healthy adolescents is adversely associated with cardiac autonomic modulation-Penn State Children Cohort. (2015) Metabolism 64(5): 626-632.

7. Vrijkotte, T.G., van den Born, B.J., Hoekstra, C.M., et al. Cardiac Autonomic Nervous System Activation and Metabolic Profile in Young Children: The ABCD Study. (2015) PLoS One 10: e0138302.

8. Willaschek, C., Meint, S., Rager, K., et al. Modified Clonidine Testing for Growth Hormone Stimulation Reveals alpha2-Adrenoreceptor Sub Sensitivity in Children with Idiopathic Growth Hormone Deficiency. (2015) PLoS One 10(10): e0137643.

9. Thayer, J.F., Lane, R.D. Claude Bernard and the heart-brain connection: further elaboration of a model of neurovisceral integration. (2009) Neurosci Biobehav Rev 33(2): 81-88.

10. Thayer, J.F., Ahs, F., Fredrikson, M., et al. A meta-analysis of heart rate variability and neuroimaging studies: implications for heart rate variability as a marker of stress and health. (2012) Neurosci Biobehav Rev 36(2): 747-756.

11. Estour, B., Galusca, B., Germain, N. Constitutional thinness and anorexia nervosa: a possible misdiagnosis? (2014) Front Endocrinol (Lausanne) $5: 175$.

12. Billman, G.E. The effect of heart rate on the heart rate variability response to autonomic interventions. (2013) Front Physiol 4:222.

13. Billman, G.E. The LF/HF ratio does not accurately measure cardiac sympatho-vagal balance. (2013) Front Physiol 4:26.

14. Glazier, D.S. Is metabolic rate a universal 'pacemaker' for biological processes? (2015) Biol Rev Camb Philos Soc 90(2): $377-407$.

15. Weitz, G., Bonnemeier, H., Sufke, S., et al. Heart rate variability and metabolic rate in healthy young adults with low birth weight. (2013) Am J Cardiovasc Dis 3(4): 239-246.

16. Scolnick, B., Mostofsky, D.I. Anorexia nervosa: a rogue hibernation? Med Hypotheses 82(2): 231-235.

17. Florant, G.L., Healy, J.E. The regulation of food intake in mammalian hibernators: a review. (2012) J Comp Physiol B 182(4): $451-467$.

18. Mrosovsky, N., Powley, T.L. Set points for body weight and fat. (1977) Behav Biol 20(2): 205-223.

19. Hastings, M., O’Neill, J.S., Maywood, E.S. Circadian clocks: regulators of endocrine and metabolic rhythms. (2007) J Endocrinol 195(2): 187-198.

20. Porges, S.W. The polyvagal theory: new insights into adaptive reactions of the autonomic nervous system. (2009) Cleve Clin J Med 76(2): S86-S90.

21. Diamond, A. Executive Functions. (2013) Annu Rev Psychol 64: 135-168.

22. Baum, P., Petroff, D., Classen, J., et al. Dysfunction of autonomic nervous system in childhood obesity: a cross-sectional study. (2013) PLoS One 8(1): e54546.

23. Pal, G.K., Pal, P., Nanda, N., et al. Sympathovagal Imbalance in Young Prehypertensives: Importance of Male-Female Difference. (2012) Am J Med Sci 345(1): 10-17.

24. Buchhorn, R., Hulpke-Wette, M., Nothroff, J., et al. Heart rate variability in infants with heart failure due to congenital heart disease: reversal of depressed heart rate variability by propranolol. (2002) Med Sci Monit 8(10): CR661-CR666.

25. Vaseghi, M., Shivkumar, K. The role of the autonomic nervous system in sudden cardiac death. (2008) Prog Cardiovasc Dis 50(6): 404-419. 
26. Ben Dov, I.Z., Kark, J.D., Ben Ishay, D., et al. Blunted heart rate dip during sleep and all-cause mortality. (2007) Arch Intern Med 167(19): 2116-2121.

27. Kaur, G., Phillips, C., Wong, K., et al. Timing is important in medication administration: a timely review of chronotherapy research. (2013) Int J Clin Pharm 35(3): 344-358.

28. Weiss, E.P., Fontana, L. Caloric restriction: powerful protection for the aging heart and vasculature. (2011) Am J Physiol Heart Circ Physiol 301(4): H1205-H1219.

29. Ahrens, W., Moreno, L.A., Marild, S., et al. Metabolic syndrome in young children: definitions and results of the IDEFICS study. (2014) Int J Obes (Lond) 38 (2): S4-S14.

30. Jarczok, M.N., Li, J., Mauss, D., et al. Heart rate variability is associated with glycemic status after controlling for components of the metabolic syndrome. (2013) Int J Cardiol 167(3): 855-861.

31. Jelinek, H.F., Md, I.H., Al Aubaidy, H., et al. Association of cardiovascular risk using non-linear heart rate variability measures with the framingham risk score in a rural population. (2013) Front Physiol 4: 186.

32. Sangun, O., Dundar, B., Kosker, M., et al. Prevalence of metabolic syndrome in obese children and adolescents using three different criteria and evaluation of risk factors. (2011) J Clin Res Pediatr Endocrinol 3(2): 70-76.

33. Rodriguez-Colon, S.M., Bixler, E.O., Li, X., et al. Obesity is associated with impaired cardiac autonomic modulation in children. (2011) Int J Pediatr Obes 6(2): 128-134.

34. Bomba, M., Corbetta, F., Gambera, A., et al. Heart rate variability in adolescents with functional hypothalamic amenorrhea and anorexia nervosa. (2014) Psychiatry Res 215: 406-409.

35. Petretta, M., Bonaduce, D., Scalfi, L., De et al. Heart rate variability as a measure of autonomic nervous system function in anorexia nervosa. (1997) Clin Cardiol 20(3): 219-224.

36. Judges, D., Beverly, S., Rio, A., et al. Clinical guidelines and enteral nutrition support: a survey of dietetic practice in the United Kingdom. (2012) Eur J Clin Nutr 66(1): 130-135.

37. Rocks, T., Pelly, F., Wilkinson, P. Nutrition therapy during initiation of refeeding in underweight children and adolescent in patients with anorexia nervosa: a systematic review of the evidence. (2014) J Acad Nutr Diet 114(6): 897-907.

38. Ayton, A.K., Azaz, A., Horrobin, D.F. A pilot open case series of ethyl-EPA supplementation in the treatment of anorexia nervosa. (2004) Prostaglandins Leukot Essent Fatty Acids 71(14): 205-209.

39. Scolnick, B., Mostofsky, D.I., Keane, R.J. Pilot study employing heart rate variability biofeedback training to decrease anxiety in patients with eating disorders. (2014) J Eat Disord 2: 17.

40. Achamrah, N., Nobis, S., Breton, J., et al. Maintaining physical activity during refeeding improves body composition, intestinal hyperpermeability and behavior in anorectic mice. (2016) Sci Rep 6: 21887.

41. Lachish, M., Stein, D., Kaplan, Z., et al. Irreversibility of cardiac autonomic dysfunction in female adolescents diagnosed with anorexia nervosa after short- and long-term weight gain. (2009) World J Biol Psychiatry 10(4): 503-511.

42. McLennan, P.L. Cardiac physiology and clinical efficacy of dietary fish oil clarified through cellular mechanisms of omega-3 polyunsaturated fatty acids. (2014) Eur J Appl Physiol 114(7): 1333-1356.

43. Widenhorn-Muller, K., Schwanda, S., Scholz, E., et al. Effect of supplementation with long-chain omega-3 polyunsaturated fatty acids on behavior and cognition in children with attention deficit/hyperactivity disorder (ADHD): a randomized placebo-controlled intervention trial. (2014) Prostaglandins Leukot Essent Fatty Acids 91(1-2): 49-60.

44. Billman, G.E. Omega-3 polyunsaturated fatty acids and cardiac rhythm: an introduction. (2012) Front Physiol 3: 457.

45. Billman, G.E. The effects of omega-3 polyunsaturated fatty acids on cardiac rhythm: a critical reassessment. (2013) Pharmacol Ther 140(1): 53-80.

46. Skilton, M.R., Pahkala, K., Viikari, J.S., et al. The association of dietary alpha-linolenic acid with blood pressure and subclinical atherosclerosis in people born small for gestational age: the Special Turku Coronary Risk Factor Intervention Project study. (2015) J Pediatr 166(5): $1252-1257$.

47. Valera, B., Muckle, G., Poirier, P., et al. Cardiac autonomic activity and blood pressure among Inuit children exposed to mercury. (2012) Neurotoxicology 33(5): 1067-1074.

48. Wenstrom, K.D. The FDA's new advice on fish: it's complicated. (2014) Am J Obstet Gynecol 211(5): 475-478.

49. Bae, S., Kim, J.H., Lim, Y.H., et al. Associations of bisphenol A exposure with heart rate variability and blood pressure. (2012) Hypertension 60(3): 786-793.

50. Pieters, N., Plusquin, M., Cox, B., et al. An epidemiological appraisal of the association between heart rate variability and particulate air pollution: a meta-analysis. (2012) Heart 98(15): 1127-1135.

51. Park, S.K., Auchincloss, A.H., O’Neill, M.S., et al. Particulate air pollution, metabolic syndrome, and heart rate variability: the multi-ethnic study of atherosclerosis (MESA). (2010) Environ Health Perspect 118(10): 1406-1411.

52. Zhang, J., Fang, S.C., Mittleman, M.A., et al. Secondhand tobacco smoke exposure and heart rate variability and inflammation among non-smoking construction workers: a repeated measures study. (2013) Environ Health 12(1): 83.

53. Lin, P.C., Kuo, S.Y., Lee, P.H., et al. Effects of internet addiction on heart rate variability in school-aged children. (2014) J Cardiovasc Nurs 29(6): 493-498.

54. Ononogbu, S., Wallenius, M., Punamaki, R.L., et al. Association between Information and Communication Technology Usage and the Quality of Sleep among School-Aged Children during a School Week. (2014) Sleep Disord 2014: 315808.

55. Tong, H., Rappold, A.G., Diaz-Sanchez, D., et al. Omega-3 fatty acid supplementation appears to attenuate particulate air pollution-induced cardiac effects and lipid changes in healthy middle-aged adults. (2012) Environ Health Perspect 120(7): 952-957.

56. Buchhorn, R., Conzelmann, A., Willaschek, C., et al. Heart rate variability and methylphenidate in children with ADHD. (2012) Atten Defic Hyperact Disord 4(2): 85-91.

57. Wood, A.R., Esko, T., Yang, J., et al. Defining the role of common variation in the genomic and biological architecture of adult human height. (2014) Nat Genet 46(11): 1173-1186.

58. Simeone, P., Alberti, S. Epigenetic heredity of human height. (2014) Physiol Rep 2(6): e12047.

59. Martins, V.J., Toledo Florencio, T.M., Grillo, L.P., et al. Long-lasting effects of undernutrition. (2011) Int J Environ Res Public Health 8(6): 
1817-1846.

60. Florencio, T.T., Ferreira, H.S., Cavalcante, J.C, et al. Food consumed does not account for the higher prevalence of obesity among stunted adults in a very-low-income population in the Northeast of Brazil (Maceio, Alagoas). (2003) Eur J Clin Nutr 57(11): 1437-1446.

61. Franco, M.C., Casarini, D.E, Carneiro-Ramos, M.S., et al. Circulating renin-angiotensin system and catecholamines in childhood: is there a role for birthweight? (2008) Clin Sci (Lond) 114(5): 375-380.

62. Fernald, L.C., Grantham-McGregor, S.M. Growth retardation is associated with changes in the stress response system and behavior in schoolaged jamaican children. (2002) J Nutr. 132(12): 3674-3679.

63. Misra, M., Klibanski, A. Endocrine consequences of anorexia nervosa. (2014) Lancet Diabetes Endocrinol 2(7): 581-592.

64. Srivastava, D., Singh, K., Misra, R., et al. Comparison of cardiac autonomic activity between malnourished and healthy children. (2012) Indian J Physiol Pharmacol 56(3): 222-228.

65. Hoffman, D.J., Sawaya, A.L., Coward, W.A., et al. Energy expenditure of stunted and nonstunted boys and girls living in the shantytowns of Sao Paulo, Brazil. (2000) Am J Clin Nutr 72(4): 1025-1031.

66. Modan-Moses, D., Yaroslavsky, A., Kochavi, B., et al. Linear growth and final height characteristics in adolescent females with anorexia nervosa. (2012) PLoS ONE 7(9): e45504.

67. Hess, S.Y., Abbeddou, S., Jimenez, E.Y., et al. Small-quantity lipid-based nutrient supplements, regardless of their zinc content, increase growth and reduce the prevalence of stunting and wasting in young burkinabe children: a cluster-randomized trial. (2015) PLoS ONE 10(3): e0122242.

68. Huffman, S.L., Harika, R.K., Eilander, A., et al. Essential fats: how do they affect growth and development of infants and young children in developing countries? A literature review. (2011) Matern Child Nutr 7(3): 44-65.

69. Ahmadi, A., Gharipour, M., Arabzadeh, G., et al. The effects of vitamin E and omega-3 PUFAs on endothelial function among adolescents with metabolic syndrome. (2014) Biomed Res Int 2014: 906019.

70. Nobili, V., Alisi, A., Della, C.C., et al. Docosahexaenoic acid for the treatment of fatty liver: randomised controlled trial in children. (2013) Nutr Metab Cardiovasc Dis 23(11): 1066-1070.

71. Moukaddem, M., Boulier, A., Apfelbaum, M., et al. Increase in diet-induced thermogenesis at the start of refeeding in severely malnourished anorexia nervosa patients. (1997) Am J Clin Nutr 66(1): 133-140.

72. Kalm, L.M., Semba, R.D. They starved so that others be better fed: remembering Ancel Keys and the Minnesota experiment. (2005) J Nutr 135(6): 1347-1352.

73. Westerterp, K.R. Metabolic adaptations to over--and underfeeding--still a matter of debate? (2013) Eur J Clin Nutr 67(5): $443-445$.

74. Ayton, A.K. Dietary polyunsaturated fatty acids and anorexia nervosa: is there a link? (2004) Nutr Neurosci 7(1): 1-12.

Online ISSN: $2377-0619$

Journal Title: International Journal Food and Nutritional Science Journal Short Name: Int J Food Nutr Sci
Ommega Online Publishers

E-mail: foodscience@ommegaonline.org

Website: www.ommegaonline.org 